DOI: $10.15393 /$ j3.art.2018.5730

UDC 517.28, 517.95, 517.926

R. Yilmazer, O. Ozturk

\title{
N-FRACTIONAL CALCULUS OPERATOR METHOD TO THE EULER EQUATION
}

\begin{abstract}
We can obtain the explicit solutions of the Euler equation by using the fractional calculus methods. So, we apply the $N$ operator method in the fractional calculus to solve this equation in this paper. We take advantage of some results of previous studies related to the fractional calculus.
\end{abstract}

Key words: fractional calculus, Euler equation, ordinary differential equation

\section{Mathematical Subject Classification: 26A33, 34A08}

1. Introduction and Preliminaries. The theory of fractional calculus (fractional integrals and fractional derivatives) has a 300 yearlong history and it has always been a subject of interest. Popularity of fractional calculus continues via important studies in the field, e.g., [1-3, 5, 8-10].

In the recent years, by making use of the following definition, properties, and characteristics of a fractional differintegral operator of order $\nu \in \mathbb{R}$, many scientists have explicitly obtained particular solutions of a number of families of homogeneous (as well as non-homogeneous) linear ordinary and partial fractional differintegral equations [6,11-14].

We find it convenient to recall here the following definition of a fractional differintegral of $y(z)$ of order $\nu$ :

Definition 1. Let $C:=\left\{C^{-}, C^{+}\right\}$. Here $C^{-}$is a contour along the cut joining the points $z$ and $-\infty+i \operatorname{Im}(z)$ that starts at $-\infty$, encircles the point $z$ once counter-clockwise, and returns to $-\infty$, and $C^{+}$is a contour along the cut joining the points $z$ and $\infty+i \operatorname{Im}(z)$ that starts at $\infty$,

(C) Petrozavodsk State University, 2018 
encircles the point $z$ once counter-clockwise, and returns to $\infty$. Let a function $y(z)$ be analytic and have no branch points inside and on $C$,

$$
y_{\nu}(z):=\frac{\Gamma(\nu+1)}{2 \pi i} \int_{C} \frac{y(\xi) d \xi}{(\xi-z)^{\nu+1}} \quad\left(\nu \notin \mathbb{Z}^{-}\right)
$$

and,

$$
y_{-n}(z):=\lim _{\nu \rightarrow-n} y_{\nu}(z) \quad\left(n \in \mathbb{Z}^{+}\right),
$$

where $\xi \neq z,-\pi \leqslant \arg (\xi-z) \leqslant \pi$ for $C^{-}$, and $0 \leqslant \arg (\xi-z) \leqslant 2 \pi$ for $C^{+}$. Then $y_{\nu}(z)(\nu>0)$ is said to be the fractional derivative of $y(z)$ of order $\nu$ and $y_{\nu}(z) \quad(\nu<0)$ is said to be the fractional integral of $y(z)$ of order $-\nu$, provided that $\left|y_{\nu}(z)\right|<\infty \quad(\nu \in \mathbb{R})[4]$.

We need the following lemmas and properties to solve ordinary and partial differential equations [4].

Lemma 1. [Linearity] Consider analytic and single-valued functions $y(z)$ and $u(z)$ and assume that $y_{\nu}(z)$ and $u_{\nu}(z)$ exist. Then

$$
[\mathbf{h} y(z)+\mathbf{k} u(z)]_{\nu}=\mathbf{h} y_{\nu}(z)+\mathbf{k} u_{\nu}(z)
$$

where $\mathbf{h}$ and $\mathbf{k}$ are constants and $\nu \in \mathbb{R}, z \in \mathbb{C}$.

Lemma 2. [Index law] Consider an analytic and single-valued function $y(z)$ and assume that $\left(y_{\rho}\right)_{\nu}(z)$ and $\left(y_{\nu}\right)_{\rho}(z)$ exist. Then

$$
\left(y_{\rho}\right)_{\nu}(z)=\left(y_{\rho+\nu}\right)(z)=\left(y_{\nu}\right)_{\rho}(z)
$$

where $\nu, \rho \in \mathbb{R}, z \in \mathbb{C}$ and $\left|\frac{\Gamma(\nu+\rho+1)}{\Gamma(\nu+1) \Gamma(\rho+1)}\right|<\infty$.

Lemma 3. [Generalized Leibniz rule] Consider analytic and single-valued functions $y(z)$ and $u(z)$ and assume that $y_{\nu}(z)$ and $u_{\nu}(z)$ exist. Then

$$
N^{\nu}[y(z) u(z)]=[y(z) u(z)]_{\nu}=\sum_{n=0}^{\infty} \frac{\Gamma(\nu+1)}{\Gamma(\nu+1-n) \Gamma(n+1)} y_{\nu-n}(z) u_{n}(z),
$$

where $\nu \in \mathbb{R}, z \in \mathbb{C}$ and $\left|\frac{\Gamma(\nu+1)}{\Gamma(\nu+1-n) \Gamma(n+1)}\right|<\infty$.

Remark. When $\lambda(\lambda \neq 0)$ is a constant,

$$
\left(e^{\lambda z}\right)_{\nu}=\lambda^{\nu} e^{\lambda z} \quad(\nu \in \mathbb{R}, z \in \mathbb{C}),
$$




$$
\begin{gathered}
\left(e^{-\lambda z}\right)_{\nu}=e^{-i \pi \nu} \lambda^{\nu} e^{-\lambda z} \quad(\nu \in \mathbb{R}, z \in \mathbb{C}), \\
\left(z^{\lambda}\right)_{\nu}=e^{-i \pi \nu} \frac{\Gamma(\nu-\lambda)}{\Gamma(-\lambda)} z^{\lambda-\nu} \quad\left(\nu \in \mathbb{R}, z \in \mathbb{C},\left|\frac{\Gamma(\nu-\lambda)}{\Gamma(-\lambda)}\right|<\infty\right) .
\end{gathered}
$$

Although our present study is similar to the Nishimoto's study in 2010 (see [7]), we have explicitly obtained the fractional solutions of the nonhomogeneous Euler equation by a theorem; also we have presented the fractional solutions of the homogeneous Euler equation and general solution of this equation via the results of this theorem. Besides, fractional solutions of the partial differential equation of the second order were also exhibited differently from Nishimoto's article.

2. $\mathbf{N}$ operator method for the Euler equation. The nonhomogeneous Euler equation of second order is reduced to a non-homogeneous linear ordinary fractional differintegral equation by means of the $N$ operator method in the following theorem. This fractional differintegral equation is a special case of the general linear ordinary fractional differintegral equation involving the polynomials $P(z ; p)$ and $Q(z ; q)$ presented by Tu et al. [12]. Thus, the fractional solutions in this article can be obtained by means of the general results proven by Tu et al. [12], when degrees of these polynomials are zero.

Theorem 1. Consider $y \in\left\{y: 0 \neq\left|y_{\nu}\right|<\infty ; \nu \in \mathbb{R}\right\}$ and $f \in\left\{f: 0 \neq\left|f_{\nu}\right|<\infty ; \nu \in \mathbb{R}\right\}$. Then the non-homogeneous Euler equation

$$
L[y, x ; \alpha, \beta]=y_{2} x^{2}+y_{1} \alpha x+y \beta=f \quad(x \neq 0),
$$

has particular solutions in fractional differintegral form, as follows:

$$
\begin{aligned}
& y=\left\{x^{-(1+\sqrt{\Delta})}\left[f_{\frac{1}{2}[(1-\alpha)+\sqrt{\Delta}]} x^{-(1-\sqrt{\Delta})}\right]_{-1}\right\}_{-1+\frac{1}{2}[(\alpha-1)-\sqrt{\Delta}]} \equiv y^{I}, \\
& y=\left\{x^{-(1-\sqrt{\Delta})}\left[f_{\frac{1}{2}[(1-\alpha)-\sqrt{\Delta}]} x^{-(1+\sqrt{\Delta})}\right]_{-1}\right\}_{-1+\frac{1}{2}[(\alpha-1)+\sqrt{\Delta}]} \equiv y^{I I}, \\
& y=\left\{x^{-1}\left[f_{\frac{1}{2}(1-\alpha)} x^{-1}\right]_{-1}\right\}_{\frac{1}{2}(\alpha-3)} \equiv y^{I I I} \text {. }
\end{aligned}
$$

Here $y_{\nu}=d^{\nu} y / d x^{\nu}$ for $\nu>0, y_{0}=y(x), f=f(x)$ and $y^{I}, y^{I I}, y^{I I I}$ are three particular solutions of equation (??) and $\alpha, \beta$ are constants.

Proof. Applying the $N$ operator method to equation (1), we have

$$
\left(y_{2} x^{2}\right)_{\nu}+\left(y_{1} \alpha x\right)_{\nu}+(y \beta)_{\nu}=f_{\nu} .
$$


Now we have

$$
\begin{aligned}
N^{\nu}\left(y_{2} x^{2}\right)=\left(y_{2} x^{2}\right)_{\nu} & =\sum_{n=0}^{2} \frac{\Gamma(\nu+1)}{n ! \Gamma(\nu+1-n)}\left(y_{2}\right)_{\nu-n}\left(x^{2}\right)_{n}= \\
& =y_{2+\nu} x^{2}+y_{1+\nu} 2 \nu x+y_{\nu} \nu(\nu-1) \\
\left(y_{1} \alpha x\right)_{\nu} & =\sum_{n=0}^{1} \frac{\Gamma(\nu+1)}{n ! \Gamma(\nu+1-n)}\left(y_{1}\right)_{\nu-n}(\alpha x)_{n}= \\
& =y_{1+\nu} \alpha x+y_{\nu} \nu \alpha
\end{aligned}
$$

and,

$$
(y \beta)_{\nu}=y_{\nu} \beta,
$$

by Lemmas 1, 2, and 3, respectively.

Therefore, we have

$$
y_{2+\nu} x^{2}+y_{1+\nu}(2 \nu+\alpha) x+y_{\nu}\left[\nu^{2}+\nu(\alpha-1)+\beta\right]=f_{\nu} .
$$

We chose $\nu$ such that

$$
\nu^{2}+\nu(\alpha-1)+\beta=0,
$$

that is,

$$
\nu=\frac{1}{2}[(1-\alpha) \pm \sqrt{\Delta}]
$$

where $\Delta=(\alpha-1)^{2}-4 \beta$.

When $\nu=\frac{1}{2}[(1-\alpha)+\sqrt{\Delta}]$ and $\Delta \neq 0$, we have

$$
y_{2+\frac{1}{2}[(1-\alpha)+\sqrt{\Delta}]} x^{2}+y_{1+\frac{1}{2}[(1-\alpha)+\sqrt{\Delta}]}(1+\sqrt{\Delta}) x=f_{\frac{1}{2}[(1-\alpha)+\sqrt{\Delta}]},
$$

from equation (7).

Set

$$
y_{1+\frac{1}{2}[(1-\alpha)+\sqrt{\Delta}]}=\phi=\phi(x) \quad\left(y=\phi_{-1+\frac{1}{2}[(\alpha-1)-\sqrt{\Delta}]}\right)
$$

we have then

$$
\phi_{1}+\phi(1+\sqrt{\Delta}) x^{-1}=f_{\frac{1}{2}[(1-\alpha)+\sqrt{\Delta}]} x^{-2},
$$

from equation (9). Then, a particular solution to this first order equation is given by

$$
\phi=x^{-(1+\sqrt{\Delta})}\left[f_{\frac{1}{2}[(1-\alpha)+\sqrt{\Delta}]} x^{-(1-\sqrt{\Delta})}\right]_{-1} .
$$


Therefore, we obtain equation (2) from (10) and (12).

When $\nu=\frac{1}{2}[(1-\alpha)-\sqrt{\Delta}]$ and $\Delta \neq 0$, we have

$$
y_{2+\frac{1}{2}[(1-\alpha)-\sqrt{\Delta}]} x^{2}+y_{1+\frac{1}{2}[(1-\alpha)-\sqrt{\Delta}]}(1-\sqrt{\Delta}) x=f_{\frac{1}{2}[(1-\alpha)-\sqrt{\Delta}]},
$$

from equation (7).

Set

$$
y_{1+\frac{1}{2}[(1-\alpha)-\sqrt{\Delta}]}=\varphi=\varphi(x) \quad\left(y=\varphi_{-1+\frac{1}{2}[(\alpha-1)+\sqrt{\Delta}]}\right) ;
$$

we have then

$$
\varphi_{1}+\varphi(1-\sqrt{\Delta}) x^{-1}=f_{\frac{1}{2}[(1-\alpha)-\sqrt{\Delta}]} x^{-2},
$$

from equation (13). Then, a particular solution to this first order equation is given by

$$
\varphi=x^{-(1-\sqrt{\Delta})}\left[f_{\frac{1}{2}[(1-\alpha)-\sqrt{\Delta}]} x^{-(1+\sqrt{\Delta})}\right]_{-1} .
$$

Therefore, we obtain equation (3) from (14) and (16). When $\Delta=0$, we have

$$
y_{2+\frac{1}{2}(1-\alpha)} x^{2}+y_{1+\frac{1}{2}(1-\alpha)} x=f_{\frac{1}{2}(1-\alpha)},
$$

from equation (7).

Set

$$
y_{1+\frac{1}{2}(1-\alpha)}=\psi=\psi(x) \quad\left(y=\psi_{\frac{1}{2}(\alpha-3)}\right),
$$

we have then

$$
\psi_{1}+\psi x^{-1}=f_{\frac{1}{2}(1-\alpha)} x^{-2},
$$

from equation (17). Then, a particular solution to this first order equation is given by

$$
\psi=x^{-1}\left[f_{\frac{1}{2}(1-\alpha)} x^{-1}\right]_{-1} .
$$

Therefore, we obtain equation (4) from (18) and (20).

Corollary 1. Consider the following homogeneous Euler equation:

$$
y_{2} x^{2}+y_{1} \alpha x+y \beta=0 \quad(x \neq 0),
$$

where $y \in\left\{y: 0 \neq\left|y_{\nu}\right|<\infty ; \nu \in \mathbb{R}\right\}$.

When $f=0$ in Theorem 1, we have

$$
\phi_{1}+\phi(1+\sqrt{\Delta}) x^{-1}=0,
$$




$$
\begin{gathered}
\varphi_{1}+\varphi(1-\sqrt{\Delta}) x^{-1}=0, \\
\psi_{1}+\psi x^{-1}=0,
\end{gathered}
$$

instead of (11), (15) and (19), respectively, and so, equation (21) has solutions of the forms:

$$
\begin{gathered}
y=h\left[x^{-(1+\sqrt{\Delta})}\right]_{-1+\frac{1}{2}[(\alpha-1)-\sqrt{\Delta}]} \equiv y^{(I)}, \\
y=h\left[x^{-(1-\sqrt{\Delta})}\right]_{-1+\frac{1}{2}[(\alpha-1)+\sqrt{\Delta}]} \equiv y^{(I I)}, \\
y=h\left[x^{-1}\right]_{\frac{1}{2}(\alpha-3)} \equiv y^{(I I I)} .
\end{gathered}
$$

where $h$ is an arbitrary constant and $y^{(I)}, y^{(I I)}, y^{(I I I)}$ are three particular solutions of equation (21).

Therefore, we obtain (25) from (22), (26) from (23), and (27) from (24).

Corollary 2. Consider $y \in\left\{y: 0 \neq\left|y_{\nu}\right|<\infty ; \nu \in \mathbb{R}\right\}$ and $f \in\left\{f: 0 \neq\left|f_{\nu}\right|<\infty ; \nu \in \mathbb{R}\right\}$. Then the non-homogeneous Euler's equation (??) is satisfied by the following equality:

$$
y=y^{I}+y^{(I)} .
$$

\section{Partial differential equations.}

Theorem 2. A partial differential equation of the second order

$$
\frac{\partial^{2} \eta}{\partial x^{2}} x^{2}+\frac{\partial \eta}{\partial x} \alpha x+\delta \eta=M \frac{\partial^{2} \eta}{\partial t^{2}}+N \frac{\partial \eta}{\partial t} \quad(\eta=\eta(x, t))
$$

has solutions of the forms:

$$
\begin{gathered}
\eta=h\left[x^{-(1+\sqrt{\Delta})}\right]_{-1+\frac{1}{2}[(\alpha-1)-\sqrt{\Delta}]} \exp \left[\frac{-N \pm \sqrt{N^{2}+4 M(\delta-\beta)}}{2 M} t\right] \\
(M N \neq 0), \\
\eta=h\left[x^{-(1-\sqrt{\Delta})}\right]_{-1+\frac{1}{2}[(\alpha-1)+\sqrt{\Delta}]} \exp \left[\frac{-N \pm \sqrt{N^{2}+4 M(\delta-\beta)}}{2 M} t\right] \\
(M N \neq 0), \\
\eta=h\left[x^{-1}\right]_{\frac{1}{2}(\alpha-3)} \exp \left[\frac{-N \pm \sqrt{N^{2}+4 M(\delta-\beta)}}{2 M} t\right] \quad(M N \neq 0),
\end{gathered}
$$




$$
\begin{aligned}
& \eta=h\left[x^{-(1+\sqrt{\Delta})}\right]_{-1+\frac{1}{2}[(\alpha-1)-\sqrt{\Delta}]} \exp \left[ \pm\left(\frac{\delta-\beta}{M}\right)^{1 / 2} t\right] \\
& (M \neq 0, N=0) \text {, } \\
& \eta=h\left[x^{-(1-\sqrt{\Delta})}\right]_{-1+\frac{1}{2}[(\alpha-1)+\sqrt{\Delta}]} \exp \left[ \pm\left(\frac{\delta-\beta}{M}\right)^{1 / 2} t\right] \\
& (M \neq 0, N=0) \text {, } \\
& \eta=h\left[x^{-1}\right]_{\frac{1}{2}(\alpha-3)} \exp \left[ \pm\left(\frac{\delta-\beta}{M}\right)^{1 / 2} t\right] \quad(M \neq 0, N=0), \\
& \eta=h\left[x^{-(1+\sqrt{\Delta})}\right]_{-1+\frac{1}{2}[(\alpha-1)-\sqrt{\Delta}]} \exp \left[\left(\frac{\delta-\beta}{N}\right) t\right] \quad(M=0, N \neq 0) \text {, } \\
& \eta=h\left[x^{-(1-\sqrt{\Delta})}\right]_{-1+\frac{1}{2}[(\alpha-1)+\sqrt{\Delta}]} \exp \left[\left(\frac{\delta-\beta}{N}\right) t\right] \quad(M=0, N \neq 0) \text {, } \\
& \eta=h\left[x^{-1}\right]_{\frac{1}{2}(\alpha-3)} \exp \left[\left(\frac{\delta-\beta}{N}\right) t\right] \quad(M=0, N \neq 0),
\end{aligned}
$$

where $\alpha, \beta$ and $\delta$ are given constants, $\beta=\delta-M \lambda^{2}-N \lambda$ with $(\alpha-1)^{2} \geqslant 4 \beta$, and $h$ is an arbitrary constant.

Proof. Let $\eta(x, t)=y(x) e^{\lambda t}(\lambda \neq 0)$ be a solution of equation (29). So,

$$
\frac{\partial \eta}{\partial t}=\lambda y e^{\lambda t}, \quad \frac{\partial^{2} \eta}{\partial t^{2}}=\lambda^{2} y e^{\lambda t}, \quad \frac{\partial \eta}{\partial x}=y_{1} e^{\lambda t}, \quad \frac{\partial^{2} \eta}{\partial x^{2}}=y_{2} e^{\lambda t}
$$

and equation (29) becomes

$$
y_{2} x^{2}+y_{1} \alpha x+y\left(\delta-M \lambda^{2}-N \lambda\right)=0 .
$$

Here, we choose $\lambda$ as $\delta-M \lambda^{2}-N \lambda=\beta$, that is

$$
\begin{gathered}
\lambda=\frac{-N \pm \sqrt{N^{2}+4 M(\delta-\beta)}}{2 M} \quad(M N \neq 0), \\
\lambda= \pm\left(\frac{\delta-\beta}{M}\right)^{1 / 2} \quad(M \neq 0, N=0), \\
\lambda=\frac{\delta-\beta}{N} \quad(M=0, N \neq 0) .
\end{gathered}
$$

Then, equation (39) becomes

$$
y_{2} x^{2}+y_{1} \alpha x+y \beta=0 .
$$


By Corollary 1, the solutions are given by

$$
\begin{gathered}
y=h\left[x^{-(1+\sqrt{\Delta})}\right]_{-1+\frac{1}{2}[(\alpha-1)-\sqrt{\Delta}]} \equiv y^{(I)}, \\
y=h\left[x^{-(1-\sqrt{\Delta})}\right]_{-1+\frac{1}{2}[(\alpha-1)+\sqrt{\Delta}]} \equiv y^{(I I)}, \\
y=h\left[x^{-1}\right]_{\frac{1}{2}(\alpha-3)} \equiv y^{(I I I)} .
\end{gathered}
$$

Thus, for $M N \neq 0$, the partial differential equation (29) has the solutions of the forms (30)-(32). Moreover, for $M \neq 0$ and $N=0$, the solutions of equation (29) are given by (33)-(35), and for $M=0$ and $N \neq 0$ by (36)-(38).

4. Conclusion. The Nishimoto fractional calculus operator method was applied to the Euler equation and fractional solutions (unlike the known ones) were obtained for this equation. This different method of solution has added originality to this paper. This study may serve as a base for new works. In addition, this method is more practical and faster than other methods, e.g., the traditional Euler method.

\section{References}

[1] Baleanu D., Diethelm K., Scalas E., Trujillo J. J. Fractional calculus models and numerical methods. World Scientific Publishing, 2012.

[2] Kilbas A. A., Srivastava H. M., Trujillo J. J. Theory and applications of fractional differential equations. Elsevier (North-Holland) Science Publishers, 2006.

[3] Miller K., Ross B. An introduction to the fractional calculus and fractional differential equations. John Wiley and Sons, 1993.

[4] Nishimoto K. Fractional calculus, Vols $I / I I / I I I / I V / V$. Descartes Press, 1984/1987/1989/1991/1996.

[5] Nishimoto K. An essence of Nishimoto's fractional calculus (Calculus of the 21 st century): Integrations and differentiations of arbitrary order. Descartes Press, 1991.

[6] Nishimoto K. Kummer's twenty-four functions and $N$-fractional calculus. Nonlinear Anal., 1997, vol. 30, pp. 1271-1282. DOI: https://doi.org/ 10.1016/S0362-546X (96)00245-3.

[7] Nishimoto K. Solutions to an Euler's equation by means of $N$-fractional calculus. J. Fract. Calc., 2010, vol. 37, pp. 29-42. 
[8] Oldham K., Spanier J. The fractional calculus; Theory and applications of differentiation and integration to arbitrary order. Academic Press, 1974.

[9] Podlubny I. Fractional differential equations: An introduction to fractional derivatives, fractional differential equations, methods of their solution and some of their applications, mathematics in science and engineering, vol. 198, Academic Press, 1999.

[10] Ross B. Fractional calculus and its applications. Springer, 1975.

[11] Srivastava H. M., Owa S., Nishimoto K. Some fractional differintegral equations. J. Math. Anal. Appl., 1985, vol. 106, pp. 360-366. DOI: https: //doi.org/10.1016/0022-247X (85)90117-9.

[12] Tu S.-T., Chyan D.-K., Srivastava H. M. Some families of ordinary and partial fractional differintegral equations. Integral Transform. Spec. Funct., 2001, vol. 11, pp. 291-302. DOI: https://doi.org/10.1080/ 10652460108819319.

[13] Yilmazer R. $N$-fractional calculus operator $N^{\mu}$ method to a modified hydrogen atom equation. Math. Commun., 2010, vol. 15, no. 2, pp. 489-501.

[14] Yilmazer R., Ozturk O. Explicit solutions of singular differential equation by means of fractional calculus operators. Abstr. Appl. Anal., 2013, vol. 2013, pp. 1-6. DOI: https://doi.org/10.1155/2013/715258.

Received June 25, 2018.

In revised form, November 08, 2018.

Accepted November 08, 2018.

Published online November 24, 2018.

Resat Yilmazer

Firat University

Elazig 23119, Turkey

E-mail: rstyilmazer@gmail.com

Okkes Ozturk

Bitlis Eren University

Bitlis 13000, Turkey

E-mail: oozturk27@gmail.com 